\title{
DIAGNÓSTICO DE LA DIMENSIÓN SOCIAL DE SOSTENIBILIDAD EN PROCESOS DE MECANIZADO MEDIANTE EL ANÁLISIS RELACIONAL GRIS
}

\section{ASSESSMENT OF SUSTAINABILITY SOCIAL DIMENSION IN MACHINING PROCESSES BY THE GREY RELATIONAL ANALYSIS}

César Ayabaca Sarria ${ }^{1}$

Carlos Vila Pastor ${ }^{2}$

1. PhD Student. Universitat Politècnica de València UPV. Departamento de Ingeniería Mecánica y de Materiales. Universitat Politècnica de València UPV. Valencia (España). E-mail: ceaysar1@doctor.upv.es. Departamento de Ingeniería Mecánica. Escuela Politécnica Nacional Quito. Ecuador. E-mail: cesar.ayabaca@epn.edu.ec

2. PhD. Profesor a tiempo completo. Universitat Politècnica de València UPV. Departamento de Ingeniería Mecánica y de Materiales, Universitat Politècnica de València. Valencia UPV (España).E-Mail: carvipas@upv.es

\section{Citación sugerida:}

Ayabaca Sarria, C. y Vila Pastor, C. (2018). Diagnóstico de la dimensión social de sostenibilidad en procesos de mecanizado mediante el análisis relacional gris. 3C Tecnología: glosas de innovación aplicadas a la pyme, 7(1), 61-78. DOI: <http://dx.doi.org/10.17993/3ctecno.2018.v7n1e25.61-78/>. 


\section{RESUMEN}

Este trabajo evalúa el desempeño de indicadores de sostenibilidad industrial desde la perspectiva de la dimensión social en operaciones de mecanizado, utilizando como herramienta de mejora el ciclo Deming: planificar, hacer, verificar y analizar. La evaluación de indicadores se realiza utilizando el análisis relacional gris. Las actividades del plan de mejora se desarrollan durante el período de análisis y se mide su impacto con el fin de fomentar una cultura de sostenibilidad en la dimensión social dentro de la empresa.

\section{ABSTRACT}

This work evaluates the performance of industrial sustainability indicators from the perspective of the social dimension in machining operations, using the Deming cycle as an improvement tool: plan, do, check and act. The evaluation of indicators is done using gray relational analysis. The activities of the improvement plan are developed during the analysis period and the impact is measured in order to promote a culture of sustainability in the social dimension within the company.

\section{PALABRAS CLAVE}

Manufactura sostenible, Indicador de sostenibilidad social, Análisis relacional gris, Arranque de viruta, Mejora de procesos.

\section{KEY WORDS}

Sustainable manufacturing, Social Sustainability indicator, Grey Relational Analysis, Chip removal, Process improvement. 


\section{INTRODUCCIÓN}

\subsection{OBJETIVOS DE DESARROLLO SOSTENIBLE}

El 25 de septiembre del 2015, se organizó la cumbre en la que 193 jefes de estado acogieron la agenda de los 17 Objetivos de Desarrollo Sostenible (ODS), que permitirán una sociedad más inclusiva y responsable con el fin de: erradicar la pobreza, proteger al planeta y asegurar la prosperidad para todos; cada objetivo tiene metas específicas, que deben alcanzarse en los próximos 15 años (United Nations, 2015a), y que debemos colaborar para conseguirlos: gobiernos, sector privado y cada uno de nosotros en las actividades que desarrollamos. En el gráfico 1. a. se muestra los objetivos planteados de desarrollo sostenible 2015-2030, los cuales son el resultado de muchos esfuerzos realizados a nivel mundial; uno de los primeros se estableció en 1983 con la Comisión Mundial sobre el Medio Ambiente y el Desarrollo (United Nations, 1987), también conocida como la Comisión Brundtland.

Esta comisión realizó reuniones alrededor del mundo y produjo un informe formal de sus hallazgos varios años después, la que se denominó "Nuestro Futuro Común". En el informe final de la Comisión, se definió el desarrollo sostenible como «el desarrollo que satisface las necesidades actuales de las personas sin comprometer la capacidad de las futuras generaciones para satisfacer las suyas», con lo que se introdujo el término en el vocabulario ambiental (United Nations, 1987).

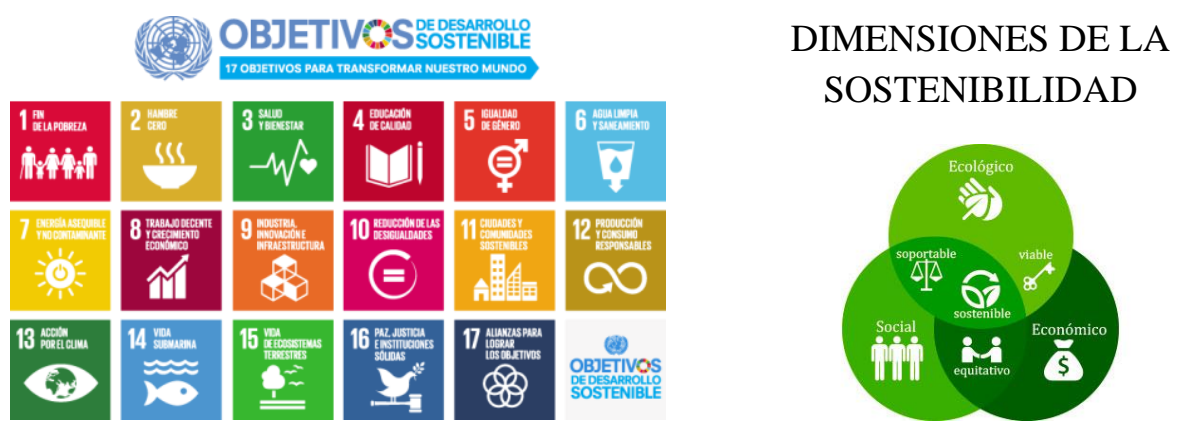

Gráfica 1. a) Objetivos de Desarrollo Sostenible 2015-2030.

b) Dimensiones de la sostenibilidad.

Fuente: a) United Nations (2015b) / b) GoConqr (2017).

Al plantear los objetivos, se establece para su análisis tres dimensiones: económica, ecológica y social, en donde la definición de sostenibilidad es aquella en la que las tres dimensiones coexisten, como se muestra en gráfico 1 b). En ese sentido es de vital importancia los esfuerzos que se realizan para minimizar el cambio climático, así como para cumplir los objetivos del desarrollo sostenible, ya que es una tarea de todos.

Desde el punto de vista industrial, los procesos requieren de un nuevo enfoque que permitan realizar este balance. En el presente trabajo se analiza la dimensión social de un taller de mecanizado, y como un proceso de mejora continua según Deming de Planear, Hacer Verificar, Actuar (PHVA), empuja la sostenibilidad en la organización. 


\subsection{PROCESOS DE REMOCIÓN DE MATERIAL}

Para la mejora de la calidad industrial se requiere de un conocimiento de los procesos industriales, en este caso analizaremos el proceso de conformado por arranque de viruta o de remoción de material (mecanizado), en el cual se definen: entradas, salidas, recursos, controles permiten medir el desempeño, así como las emisiones generadas, que son evaluadas en el contexto de sostenibilidad. Un proceso de mecanizado estándar detallado se muestra en el gráfico 2 (Helu, Dornfeld, 2013) en donde:

- FUNCIÓN: remoción de material, o arranque de viruta. Los más comunes en los talleres industriales son el torneado, fresado, taladrado y rectificado, en equipos manuales, automáticos o de control numérico.

- ENTRADAS: materiales a ser mecanizados, herramientas, fluido de corte y facilidades industriales del proceso.

- SALIDAS: la parte mecanizada que debe ser limpiada, ya que del proceso generalmente utiliza fluido de corte y requiere una limpieza final. El proceso genera material removido en forma de viruta, así como el líquido de corte, herramienta desgastada y emisiones al ambiente.

- RECURSOS: el consumo de energía en sus diferentes en las diferentes etapas, por ejemplo: energía consumida por la máquina herramienta, energía en la preparación del fluido de corte (aceite de corte, agua, aditivos, aire, etc.), así como el requerimiento de aire comprimido para la operación de sistemas auxiliares

- CONTROLES: o indicadores de proceso, que son los que permiten medir la eficiencia y la eficacia del proceso. Para la evaluación de los indicadores la perspectiva de sostenibilidad nos permitirá valorarlos desde las dimensiones económicas, ambientales y sociales.

- EMISIONES: o conocidas como desperdicios o desechos que se generan por la realización del proceso productivo, pudiendo ser físicos o químicos, como emisiones al ambiente, polución del aire, desperdicios, viruta, fluido de corte consumido, aguas residuales etc.

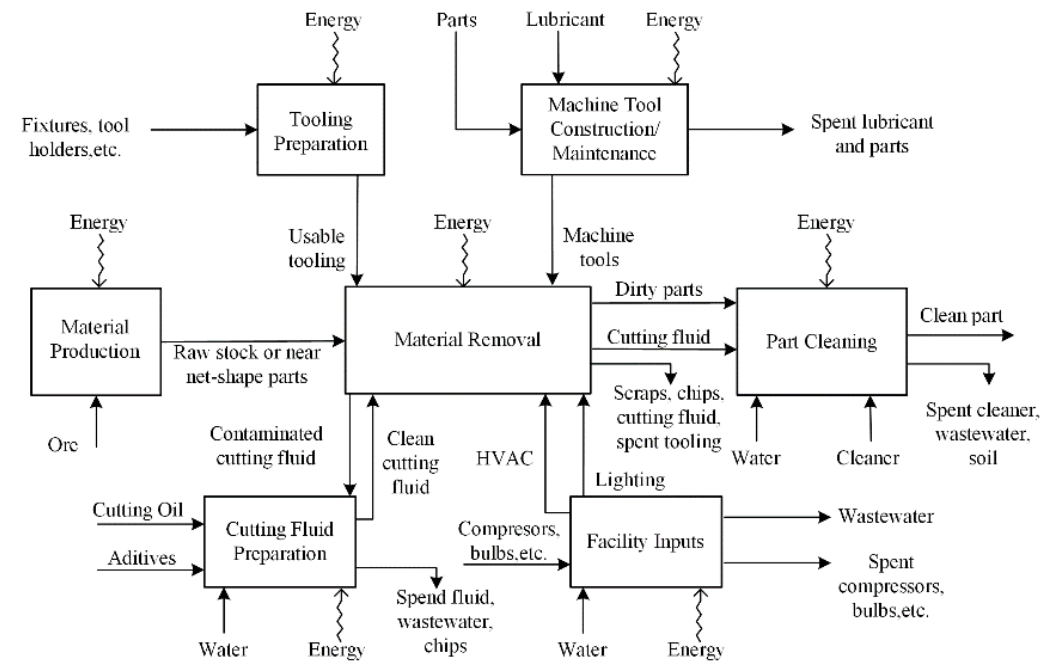

Gráfica 2. Proceso de mecanizado estándar.

Fuente: Helu (2013). 


\subsection{INDICADORES SOSTENIBLES EN LAS DIMENSIONES ECONÓMICA- SOCIAL-AMBIENTAL}

Para un mejor entendimiento de los indicadores que se utilizan, mencionaremos algunos marcos de referencia considerados para las evaluaciones de sostenibilidad, entre las más representativas tenemos:

a) Naciones Unidas (United Nations), Objetivos del desarrollo sostenible.

b) ISO 26000:2010 Responsabilidad Social.

c) ISO 14031:2013 Gestión ambiental-Evaluación del desempeño.

d) Repositorio de Indicadores de fabricación Sostenible (SMIR) del NIST.

e) Pacto global de naciones unidas (Global Compact).

f) Guía para multinacionales de OCDE. (Global Reporting Initiative).

Recientemente se han presentado propuestas de indicadores sostenibles en varios estudios, de entre los cuales, la clasificación de indicadores para una firma manufacturera presentada por Bhanot ha sido selecciona para evaluar los indicadores de sostenibilidad (Bhanot, Venkateswara, Deshmukh, 2016a), los cuales se describen a continuación:

a) En la dimensión económica: costo de producción, calidad de corte, tasa de producción, gestión de procesos.

b) En la dimensión ambiental: Intensidad del consumo de agua, intensidad energética, materiales utilizados, gestión de residuos, regulaciones ambientales.

c) En la dimensión social: salud del trabajador, seguridad del trabajador, relaciones laborales, capacitación y educación: horas de capacitación por operador; nivel de habilidad requerido.

\subsection{MEJORA CONTINUA DE PROCESOS}

El ciclo de mejora continua PHVA Planear-Hacer-Verificar-Actuar es el utilizado en los círculos de calidad y el más difundido en las normas ISO; como se ilustra en el Gráfico 3 a), para lo cual el proceso de mejora de la calidad requiere dar varias vueltas al ciclo PHVA, lo cual se representa como un conjunto de círculos subiendo una pendiente.
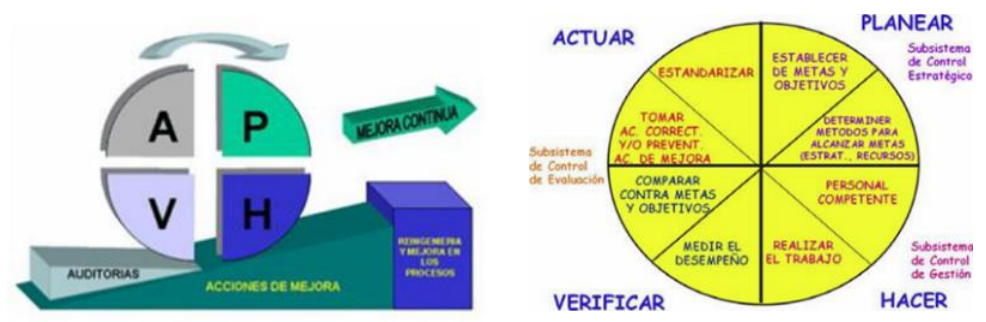

Gráfico 3. a) Ciclo PHVA. b) Gerencia de la calidad Deming.

Fuente: a) (Securitijefer, 2010); b) (Emprendices (2010).

La cuña representa a los sistemas de calidad que se requieren para que el ciclo se mantenga. Complementando esta propuesta, Miyauchi (ITESM, 2013), propone un gráfico explicativo ampliado para el control de procesos del Ciclo PHVA el que se describe a continuación en el gráfico 4, en el cual 
se destaca el ciclo de mantenimiento, el ciclo de mejoramiento y el ciclo de corrección que son parte de lo que se conoce como la Gestión de la Calidad.

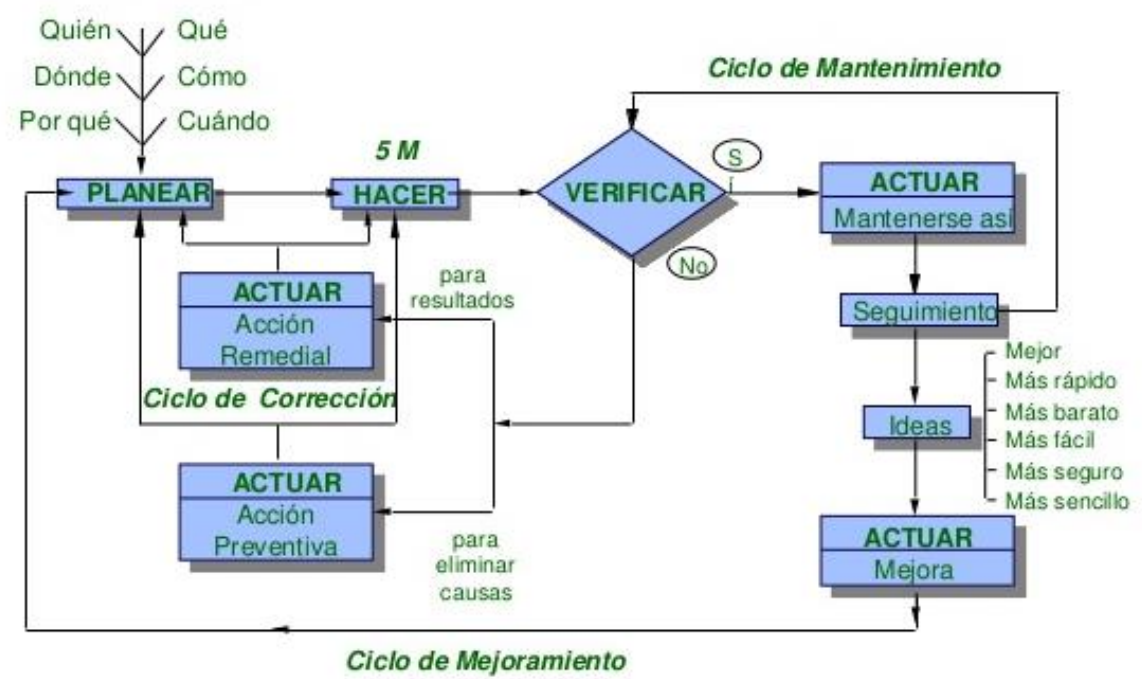

Gráfico 4. Gestión de la Calidad definida por el Dr. Miyauchi.

Fuente: (ITESM, 2013).

\section{METODOLOGÍA}

\subsection{DIAGNÓSTICO DE SOSTENIBILIDAD DE LA DIMENSIÓN SOCIAL POR EL MÉTODO GREY RELATIONAL ANALYSIS (GRA)}

La metodología por seguir se describe en la Tabla 1, en donde para cada etapa del ciclo de mejora continua, se define los pasos y actividades a realizar.

\begin{tabular}{ccl} 
ETAPA & PASO & \multicolumn{1}{c}{ ACTIVIDADES A REALIZAR } \\
\hline \multirow{3}{*}{ Planear } & 1 & Definir el Proyecto de mejora \\
& 2 & Describir la situación Actual \\
& 3 & Analizar hechos y datos \\
& 4 & Establecer acciones \\
\cline { 2 - 3 } Hacer & 5 & Ejecutar las acciones Establecidas \\
\cline { 2 - 3 } Verificar & 6 & Verificar los resultados \\
\cline { 2 - 3 } Actuar & 7 & Estandarizar \\
& 8 & Documentar y definir nuevos planes de mejora \\
\hline
\end{tabular}

Tabla 1. Etapas del Ciclo PHVA para mejora de sostenibilidad.

Fuente: Elaboración propia.

- Paso 1: Se define el proyecto de mejora de sostenibilidad: en el presente análisis es de nuestro interés solamente la dimensión social, dejando la dimensión económica y ambiental para análisis futuros.

- Paso 2: Se describe la situación actual de sostenibilidad de la empresa en la dimensión social: se realiza una evaluación inicial utilizando los indicadores de sostenibilidad de la dimensión social descritos en la bibliografía a los colaboradores de la empresa (trabajadores). 


\section{$\left.{ }^{3} \mathrm{c}\right)$ tecnología}

- Paso 3: Se analiza los hechos y datos: utilizando el método del Análisis Relacional Gris (Grey Relational Analysis, GRA), con el resultado de la evaluación inicial se calculan los indicadores iniciales para el plan de mejora.

- Paso 4: Establecer las acciones: Se elabora el plan de mejora considerando las herramientas del ciclo de mejora continua en donde se define: el problema a mejorar, objetivos, metas, indicadores de sostenibilidad, estrategias, actividades, responsable y periodo en el que se van a realizar.

- Paso 5: Ejecutar las acciones establecidas: Se ejecuta las acciones identificadas en el plan de mejora. En el desarrollo participa todo el personal, colaboradores, supervisores con el apoyo de los directivos de la empresa; los auditores externos registran el avance y la planificación para su posterior evaluación.

- Paso 6: Verificar los resultados: en este paso se analiza la efectividad de las acciones implementadas, teniendo en consideración el plan propuesto.

- Paso 7: Estandarizar: Se genera gráficos de control, de los indicadores de la dimensión social para su posterior registro y análisis.

- Paso 8: Documentar y definir nuevos planes de mejora: se realiza un seguimiento a los resultados obtenidos y se comienza un nuevo ciclo; la evaluación final será el punto de partida para un nuevo ciclo PHVA.

\subsection{INDICADORES PARA LA DIMENSIÓN SOCIAL}

Para la formulación de indicadores de la dimensión social, se utiliza los recomendados en estudios de empresas similares realizados por Bhanot. La evaluación se ha desarrollado a los 5 colaboradores que trabajan en el área de manufactura, de tal manera que la calificación se realiza en una escala de 1 a 5 siendo la evaluación más baja $1=$ nulo, y la más alta $5=$ completamente satisfactorio. Esta evaluación se realiza por tres agentes distintos: a) los mismos trabajadores, b) los supervisores con respecto a los trabajadores y c) una auditoría de terceros en algunos aspectos, en donde se usa el Análisis Relacional Gris según Bhanot (Bhanot, Venkateswara, Deshmukh, 2016b). Se generan tres grupos de indicadores para evaluar la dimensión social.

En un primer grupo, los indicadores clasificados por los trabajadores se basan en diversos temas, como los problemas de gestión, el entorno laboral y las cuestiones gubernamentales, de los cuales se ha seleccionado los siguientes:

1. Soporte de la alta gerencia en varios asuntos.

2. Nivel de satisfacción laboral (salario, incentivos y carga de trabajo).

3. Ambiente de trabajo propicio (condiciones de trabajo y diseño ergonómico del espacio de trabajo).

4. Alcance del apoyo del gobierno:

a) Conciencia sobre iniciativas de manufactura sustentable.

b) Actualización tecnológica a través de centros de capacitación. Apoyo financiero (en forma de préstamos, incentivos fiscales, etc.).

En un segundo grupo, los indicadores son clasificados por el supervisor para todos los trabajadores en relación con diversos temas, como el desempeño, el comportamiento, entre los que destacan:

1. Productividad del trabajador. (que tan eficiente rápido se fabrican los productos). 


\section{$\left.{ }^{3} \mathrm{c}\right)$ tecnología}

2. Relaciones cordiales con otros trabajadores.

3. Nivel de habilidad del trabajador.

4. Flexibilidad a la rotación laboral.

5. Puntualidad para trabajar.

En un tercer grupo, los indicadores son calificados por auditores externos, con respecto a los problemas de los trabajadores, y según el grado en que los trabajadores cumplen con los requisitos normativos nacionales e internacionales dados por el gobierno.

1. Requisitos de calidad y funcionalidad de los productos.

2. Prácticas de manejo de desechos.

3. Prácticas de conservación de energía.

4. Seguridad operacional.

5. Salud e higiene del personal (incluidas las prácticas seguidas por los trabajadores y el impacto de los refrigerantes y lubricantes).

Por lo tanto, el siguiente paso en el análisis es la aplicación de GRA para obtener un índice de rendimiento adecuado el que se explica a continuación.

\subsection{ANÁLISIS RELACIONAL GRIS (GREY RELATIONAL ANALYSIS, GRA)}

En los trabajos presentados sobre evaluación de indicadores, se menciona que la teoría de sistemas grises permite tratar con información incompleta e incierta (Bhanot, 2016a). En esta teoría, el sistema blanco representa la presencia de información completa y, por otro lado, el sistema negro representa la ausencia de información. Sin embargo, los sistemas grises representan el nivel intermedio de información.

El Análisis Relacional Gris es utilizado en la evaluación de los procesos de mecanizado; las investigaciones miden el efecto de estos parámetros en las dimensiones económica, ambiental y social (Wang, Lu, Li X.X, and Li. W.D., 2015) así como son los presentados por Bhanot, donde se explica el procedimiento de aplicación mediante los siguientes pasos:

- Preparación de los datos Es importante normalizar los datos en el rango de 0-1 ya que, dependiendo de la naturaleza de la variable, un valor cercano a 1 puede ser indicación de un mejor rendimiento, es decir "alto es lo mejor" como, por ejemplo, en procesos de mecanizado: la tasa de remoción de material, el tiempo de vida de la herramienta; mientras que para otras variables " inferior es lo mejor ", por ejemplo. rugosidad superficial, temperatura de corte y costo de producción. Considerando las ' $m$ ' alternativas y ' $n$ ' atributos de respuesta para un problema multiobjetivo, la i-ésima alternativa se puede expresar como $Y_{i}=\left(y_{i 1}, y_{i 2}, \ldots, y_{i j}, \ldots ., y_{i n}\right)$, en donde $y_{i j}$ representa el valor del atributo $j$ para la alternativa $i$. Sin embargo, el termino $Y_{i}$ se puede traducir a la secuencia de comparabilidad $X_{i}=\left(x_{i 1}, x_{i 2}, \ldots, x_{i j}, \ldots, x_{i n}\right)$, empleando las siguientes ecuaciones. El procedimiento de normalización de datos para los criterios de "alto es lo mejor" se muestra a continuación: 


$$
X_{i j}=\frac{y_{i j}-\operatorname{Min}\left(y_{i j}, i=1,2, \ldots m\right)}{\operatorname{Max}\left(y_{i j}, i=1,2, \ldots, m\right)-\operatorname{Min}\left(y_{i j}, i=1,2, \ldots m\right)}
$$

Sin embargo, la normalización de datos para el criterio de" menor es lo mejor " se muestra a continuación

$$
X_{i j}=\frac{\operatorname{Max}\left(y_{i j}, i=1,2, \ldots m\right)-y_{i j}}{\operatorname{Max}\left(y_{i j}, i=1,2, \ldots, m\right)-\operatorname{Min}\left(y_{i j} i=1,2, \ldots m\right)}
$$

- Determinación de coeficientes relacionales grises. Para determinar los coeficientes relacionales grises, una secuencia de referencia $X_{0}$ se define como $\left(x_{01}, x_{02}, \ldots, x_{0 j}, \ldots, x_{0 n}\right)=(1,1,1, \ldots, 1, \ldots, 1)$. Este coeficiente tiende a determinar la cercanía entre secuencia de comparabilidad y la serie de referencia utilizando la siguiente fórmula:

$$
\gamma\left(x_{0 j}, x_{i j}\right)=\frac{\Delta_{\min }+\zeta \Delta_{\max }}{\Delta_{i j}+\zeta \Delta_{\max }}
$$

Para $i=1,2, \ldots, m$ y $j=1,2, \ldots, n$

Donde:

$$
\begin{aligned}
& \gamma\left(x_{0 j}, x_{i j}\right) \text { es el coeficiente relacional gris entre } x_{0 j} \text { y } x_{i j} \\
& \Delta_{i j}=\left|x_{0 i}-x_{i j}\right| \\
& \Delta_{\min }=\operatorname{Min}\left(\Delta_{i j}, i=1,2, \ldots, m ; j=1,2, \ldots, n\right) \\
& \Delta_{\max }=\operatorname{Max}\left(\Delta_{i j}, i=1,2, \ldots, m ; j=1,2, \ldots, n\right) \\
& \zeta \text { es el coeficiente distintivo y } \zeta \epsilon\{0,1\}
\end{aligned}
$$

- Cálculo de Grados Relacionales Grises. La calificación de Grado Relacional Gris (GRG) puede calcularse asignando ponderaciones adecuadas a los coeficientes relacionales grises de cada atributo de respuesta de la siguiente manera:

$$
\Gamma\left(x_{0}, x_{i}\right)=\sum_{j=1}^{n} w_{j} \gamma\left(x_{0 j}, x_{i j}\right) \text { para } i=1,2, \ldots m
$$

Sin embargo, en el caso de la dimensión social, la escala Likert se ha aplicado en una escala de 1-5 (Wu, 2007). En donde la calificación de 5 indicaría completamente satisfactorio y 1 indicaría deficiente. El índice GRG, es el promedio de las evaluaciones a cada uno de los colaboradores de la empresa analizada, que trabajan en el área del taller de manufactura. 


\section{RESULTADOS}

Se utiliza la metodología que describe en el ciclo PHVA de la Tabla 1, en donde para cada etapa del ciclo de mejora continua se desarrolla específicamente para cada paso:

PASO 1. Definir el proyecto de mejora de sostenibilidad: El proyecto busca mejorar los aspectos de dimensión social y como apalancan a las dimensiones económica y ambiental dentro de la empresa. Para ello durante el periodo 2016 se realizarán varias actividades descritas en el plan con el fin de medir y mejorar la dimensión social.

PASO 2. Describir la situación Actual: Como en toda empresa, hay cambios que generan oportunidades de mejora; mencionaremos las más importantes al inicio de la presente evaluación que se muestra en la Tabla 3 y Tabla 4

Se realiza la medición de indicadores de sostenibilidad que será la línea base del presente análisis. En la tabla 2 se muestra las calificaciones iniciales. Los valores promedios son redondeados al entero superior para facilitar el análisis. En la Tabla 3 se muestra el cálculo del índice GRG para cada indicador, utilizando el Análisis relacional gris; cabe mencionar algunas consideraciones que se tenían definidas, cuando se realiza esta evaluación inicial; entre las más representativas mencionamos las siguientes:

a) Existen cambios en el personal de la empresa: directivos y mandos medios, lo que ha permitido contar con personal joven y capacitado para actividades de servicio.

b) Las evaluaciones que se realizaban se enfocaban mucho en el cumplimiento de actividades y objetivos económicos, dejando de lado los aspectos ambientales y sociales.

c) Se refuerza la implementación de planes de mantenimiento correctivo a los equipos del taller en los que han participado los colaboradores ha permitido tener un conocimiento mayor del funcionamiento y operación de estos. 


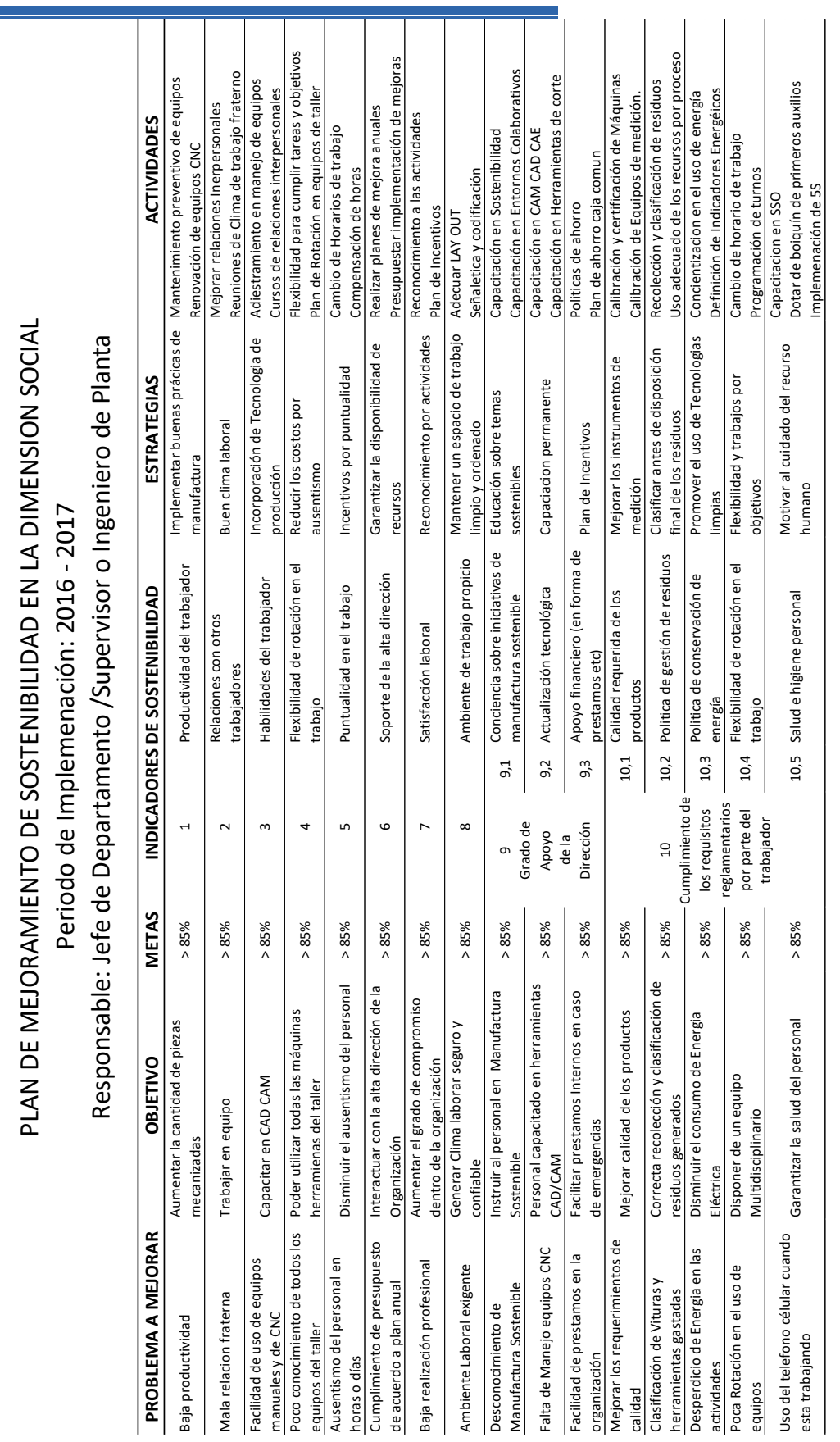

Tabla 2. Plan de Mejoramiento de Sostenibilidad en la Dimensión social. Fuente: elaboración propia. 


\begin{tabular}{|c|c|c|c|c|c|c|c|}
\hline$\#$ & $\begin{array}{l}\text { INDICADORES SOCIALES } \\
\text { EVALUACION INICIAL }\end{array}$ & $\begin{array}{l}\text { Colaborador } \\
\quad \# 1\end{array}$ & $\begin{array}{c}\text { Colaborador } \\
\quad \# 2\end{array}$ & $\begin{array}{c}\text { Colaborador } \\
\qquad \# 3\end{array}$ & $\begin{array}{c}\text { Colaborador } \\
\quad \# 4\end{array}$ & $\begin{array}{c}\text { Colaborador } \\
\quad \# 5\end{array}$ & Calificado por: \\
\hline \multirow{3}{*}{1} & \multirow{3}{*}{ Productividad del trabajador } & 4,00 & 4,00 & 4,00 & 5,00 & 4,00 & \multirow{2}{*}{$\begin{array}{c}\text { Jefe de Departamento } \\
\text { Ingeniero } 1\end{array}$} \\
\hline & & 3,00 & 4,00 & 4,00 & 4,00 & 3,00 & \\
\hline & & 4,00 & 4,00 & 4,00 & 5,00 & 4,00 & Promedio \\
\hline \multirow{3}{*}{2} & \multirow{3}{*}{$\begin{array}{l}\text { Relaciones con otros } \\
\text { trabajadores }\end{array}$} & 4,00 & 5,00 & 5,00 & 5,00 & 4,00 & \multirow{2}{*}{$\begin{array}{c}\text { Jefe de Departamento } \\
\text { Ingeniero } 1\end{array}$} \\
\hline & & 3,00 & 5,00 & 4,00 & 4,00 & 4,00 & \\
\hline & & 4,00 & 5,00 & 5,00 & 5,00 & 4,00 & Promedio \\
\hline \multirow{3}{*}{3} & \multirow{3}{*}{ Habilidades del trabajador } & 4,00 & 4,00 & 4,00 & 5,00 & 4,00 & \multirow{2}{*}{$\begin{array}{c}\text { Jefe de Departamento } \\
\text { Ingeniero } 1\end{array}$} \\
\hline & & 3,00 & 3,00 & 4,00 & 5,00 & 3,00 & \\
\hline & & 4,00 & 4,00 & 4,00 & 5,00 & 4,00 & Promedio \\
\hline \multirow{3}{*}{4} & \multirow{3}{*}{$\begin{array}{l}\text { Flexibilidad de rotación en el } \\
\text { trabajo }\end{array}$} & 3,00 & 5,00 & 3,00 & 4,00 & 3,00 & \multirow{2}{*}{$\begin{array}{c}\text { Jefe de Departamento } \\
\text { Ingeniero } 1\end{array}$} \\
\hline & & 3,00 & 3,00 & 4,00 & 4,00 & 3,00 & \\
\hline & & 3,00 & 5,00 & 4,00 & 4,00 & 3,00 & Promedio \\
\hline \multirow{3}{*}{5} & \multirow{3}{*}{ Puntualidad en el trabajo } & 4,00 & 3,00 & 4,00 & 4,00 & 4,00 & \multirow{2}{*}{$\begin{array}{c}\text { Jefe de Departamento } \\
\text { Ingeniero } 1\end{array}$} \\
\hline & & 3,00 & 3,00 & 2,00 & 4,00 & 4,00 & \\
\hline & & 4,00 & 3,00 & 4,00 & 4,00 & 4,00 & \multirow{8}{*}{$\begin{array}{l}\text { Calificado por los } \\
\text { mismos trabajadores }\end{array}$} \\
\hline 6 & Soporte de la alta dirección & 3,00 & 4,00 & 3,00 & 4,00 & 4,00 & \\
\hline 7 & Satisfacción laboral & 4,00 & 4,00 & 4,00 & 4,00 & 4,00 & \\
\hline 8 & Ambiente de trabajo propicio & 3,00 & 4,00 & 4,00 & 3,00 & 3,00 & \\
\hline 9 & \multicolumn{6}{|l|}{ Grado de apoyo de la dirección } & \\
\hline 9,1 & $\begin{array}{l}\text { Conciencia sobre iniciativas } \\
\text { de manufactura sostenible }\end{array}$ & 2,00 & 3,00 & 2,00 & 4,00 & 3,00 & \\
\hline 9,2 & Actualización tecnológica & 3,00 & 3,00 & 4,00 & 4,00 & 3,00 & \\
\hline 9,3 & $\begin{array}{l}\text { Apoyo financiero (en forma } \\
\text { de prestamos etc) }\end{array}$ & 3,00 & 3,00 & 2,00 & 3,00 & 3,00 & \\
\hline
\end{tabular}

Cumplimiento de los

\begin{tabular}{|c|c|c|c|c|c|c|c|}
\hline 10 & $\begin{array}{l}\text { Cumplimiento de los } \\
\text { requisitos reglamentarios por } \\
\text { parte del trabajador: }\end{array}$ & $\begin{array}{c}\text { Colaborador } \\
\quad \# 1\end{array}$ & $\begin{array}{c}\text { Colaborador } \\
\quad \# 2\end{array}$ & $\begin{array}{c}\text { Colaborador } \\
\quad \# 3\end{array}$ & $\begin{array}{c}\text { Colaborador } \\
\quad \# 4\end{array}$ & $\begin{array}{c}\text { Colaborador } \\
\quad \# 5\end{array}$ & Calificado por: \\
\hline \multirow{3}{*}{10,1} & \multirow{3}{*}{$\begin{array}{l}\text { Calidad requerida de los } \\
\text { productos }\end{array}$} & 3,00 & 3,00 & 4,00 & 5,00 & 5,00 & Auditor 1 \\
\hline & & 4,00 & 4,00 & 5,00 & 5,00 & 5,00 & Auditor 2 \\
\hline & & 4,00 & 4,00 & 5,00 & 5,00 & 5,00 & Promedio \\
\hline \multirow{3}{*}{10,2} & \multirow{3}{*}{$\begin{array}{l}\text { Politica de gestión de } \\
\text { residuos }\end{array}$} & 3,00 & 3,00 & 3,00 & 4,00 & 3,00 & Auditor 1 \\
\hline & & 4,00 & 2,00 & 3,00 & 4,00 & 4,00 & Auditor 2 \\
\hline & & 4,00 & 3,00 & 3,00 & 4,00 & 4,00 & Promedio \\
\hline \multirow{3}{*}{10,3} & \multirow{3}{*}{$\begin{array}{l}\text { Politica de conservación de } \\
\text { energía }\end{array}$} & 4,00 & 2,00 & 4,00 & 4,00 & 3,00 & Auditor 1 \\
\hline & & 3,00 & 2,00 & 3,00 & 4,00 & 3,00 & Auditor 2 \\
\hline & & 4,00 & 2,00 & 4,00 & 4,00 & 3,00 & Promedio \\
\hline \multirow{3}{*}{10,4} & \multirow{3}{*}{$\begin{array}{l}\text { Flexibilidad de rotación en el } \\
\text { trabajo }\end{array}$} & 3,00 & 2,00 & 4,00 & 4,00 & 3,00 & Auditor 1 \\
\hline & & 4,00 & 3,00 & 3,00 & 5,00 & 4,00 & Auditor 2 \\
\hline & & 4,00 & 3,00 & 4,00 & 5,00 & 4,00 & Promedio \\
\hline \multirow{3}{*}{10,5} & \multirow{3}{*}{ Salud e higiene personal } & 3,00 & 3,00 & 3,00 & 4,00 & 3,00 & Auditor 1 \\
\hline & & 4,00 & 5,00 & 5,00 & 3,00 & 4,00 & Auditor 2 \\
\hline & & 4,00 & 5,00 & 5,00 & 4,00 & 4,00 & Promedio \\
\hline
\end{tabular}

Tabla 3. Evaluación Inicial de Indicadores de sostenibilidad. -Dimensión Social. Fuente: elaboración propia, basada en Bhanot (2016a).

PASO 3. Analizar los hechos y los datos: La evaluación obtenida, permite ver que hay oportunidades de mejora, identificando la actualización tecnológica como el punto más débil. Se realizan reuniones de trabajo en el departamento, en las cuales mediante una lluvia de ideas se establece los problemas que están afectando el desempeño de los trabajadores, el cual se resume y se analiza para formular un plan de mejora. 


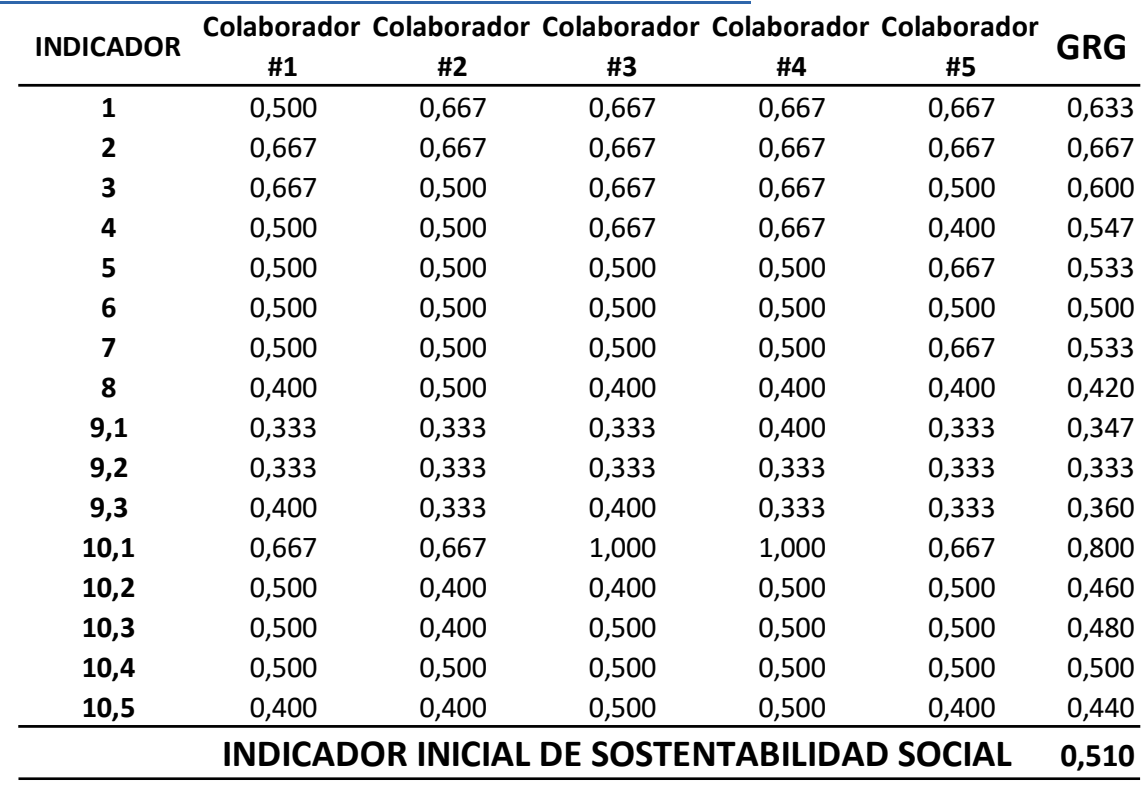

Tabla 4. Índice GRG - Evaluación Inicial de Indicadores de sostenibilidad. Dimensión Social. Fuente: Elaboración propia, basada en Bhanot (2016a).

PASO 4. Establecer acciones y planes de Mejora: Se desarrolla el plan de mejoramiento de sostenibilidad que se muestra en la Tabla 2; en donde se identifica los problemas y actividades para un periodo de un año en el que se implementan.

PASO 5. Ejecutar las Acciones establecidas: Para la ejecución se requiere la mayor colaboración de todos, se realizan actividades técnicas, de mejora de ambiente, de instalaciones y de relaciones interpersonales entre los colaboradores. El plan de capacitación en tecnologías CAD/CAM requirió de un notable esfuerzo de los participantes y un entrenamiento en los equipos CNC.

PASO 6. Verificar los resultados: Se realiza una evaluación al terminar el periodo del plan, identificando la implementación de las acciones. En la Tabla 5 se muestra la evaluación fina de sostenibilidad. En la Tabla 6 los índices GRC, de la aplicación del Análisis relacional gris.

PASO 7. Estandarizar: Con los datos de la evaluación inicial y en este primer ciclo de mejora continua se establece el cuadro de mando integral para los indicadores de la dimensión social. De acuerdo con las recomendaciones de los auditores externos una me del $85 \%$ de cumplimiento sería considerada como un objetivo que la empresa en el mediano plazo, como se muestra en la Tabla 7. En la Tabla 8 se muestra una representación gráfica de la evolución de los indicadores, Dashboard, la cual es de ayuda visual para el personal de la empresa. 


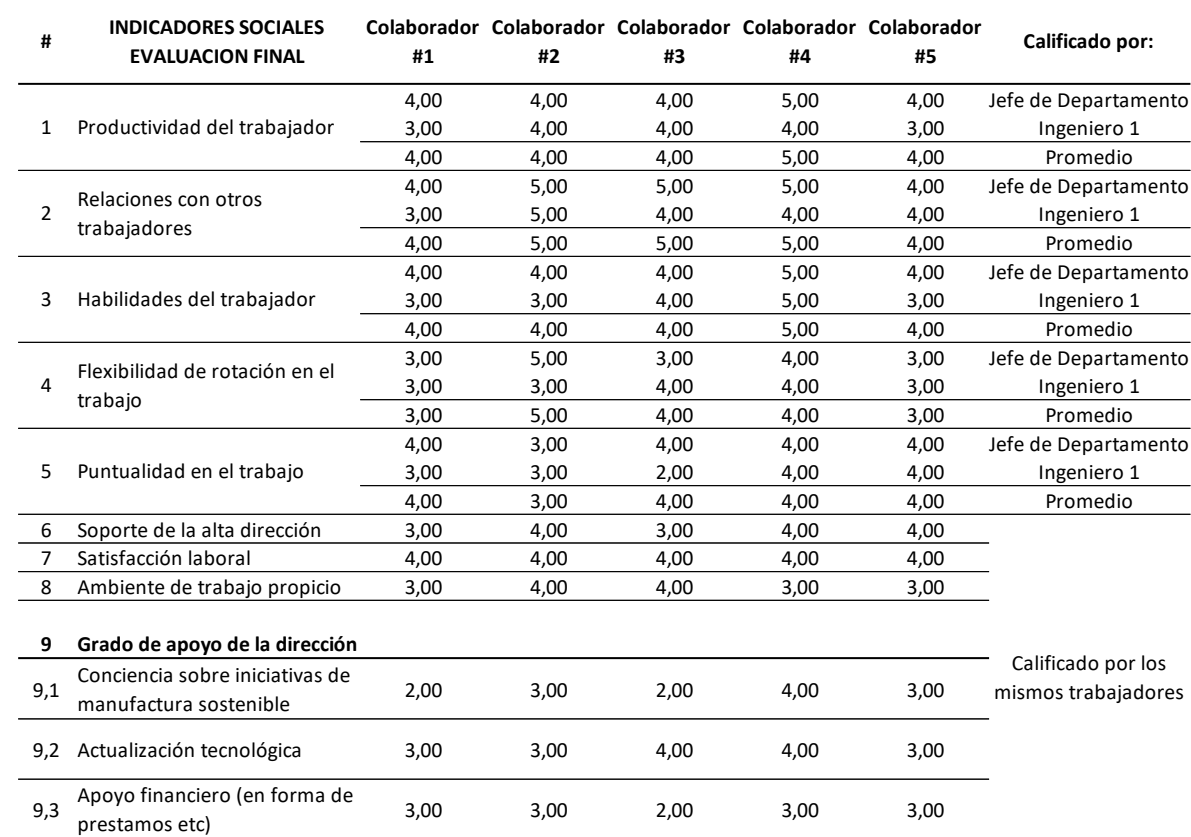

Cumplimiento de los

10 requisitos reglamentarios por Colaborador Colaborador Colaborador Colaborador Colaborador Calificado por:

\begin{tabular}{|c|c|c|c|c|c|c|c|}
\hline & parte del trabajador: & $\# 1$ & \#2 & $\# 3$ & $\# 4$ & $\# 5$ & \\
\hline \multirow{3}{*}{10,1} & \multirow{3}{*}{$\begin{array}{l}\text { Calidad requerida de los } \\
\text { productos }\end{array}$} & 3,00 & 3,00 & 4,00 & 5,00 & 5,00 & Auditor 1 \\
\hline & & 4,00 & 4,00 & 5,00 & 5,00 & 5,00 & Auditor 2 \\
\hline & & 4,00 & 4,00 & 5,00 & 5,00 & 5,00 & Promedio \\
\hline \multirow{3}{*}{10,2} & \multirow{3}{*}{ Politica de gestión de residuos } & 3,00 & 3,00 & 3,00 & 4,00 & 3,00 & Auditor 1 \\
\hline & & 4,00 & 2,00 & 3,00 & 4,00 & 4,00 & Auditor 2 \\
\hline & & 4,00 & 3,00 & 3,00 & 4,00 & 4,00 & Promedio \\
\hline \multirow{3}{*}{10,3} & \multirow{3}{*}{$\begin{array}{l}\text { Politica de conservación de } \\
\text { energía }\end{array}$} & 4,00 & 2,00 & 4,00 & 4,00 & 3,00 & Auditor 1 \\
\hline & & 3,00 & 2,00 & 3,00 & 4,00 & 3,00 & Auditor 2 \\
\hline & & 4,00 & 2,00 & 4,00 & 4,00 & 3,00 & Promedio \\
\hline \multirow{3}{*}{10,4} & \multirow{3}{*}{$\begin{array}{l}\text { Flexibilidad de rotación en el } \\
\text { trabajo }\end{array}$} & 3,00 & 2,00 & 4,00 & 4,00 & 3,00 & Auditor 1 \\
\hline & & 4,00 & 3,00 & 3,00 & 5,00 & 4,00 & Auditor 2 \\
\hline & & 4,00 & 3,00 & 4,00 & 5,00 & 4,00 & Promedio \\
\hline \multirow{3}{*}{10,5} & \multirow{3}{*}{ Salud e higiene personal } & 3,00 & 3,00 & 3,00 & 4,00 & 3,00 & Auditor 1 \\
\hline & & 4,00 & 5,00 & 5,00 & 3,00 & 4,00 & Auditor 2 \\
\hline & & 4,00 & 5,00 & 5,00 & 4,00 & 4,00 & Promedio \\
\hline
\end{tabular}

Tabla 5. Evaluación Final de Indicadores de sostenibilidad. Dimensión Social. Fuente: elaboración propia, basada en Bhanot (2016a).

\begin{tabular}{|c|c|c|c|c|c|c|}
\hline \multirow{2}{*}{ INDICADOR } & \multicolumn{5}{|c|}{ Colaborador Colaborador Colaborador Colaborador Colaborador } & \multirow{2}{*}{ GRG } \\
\hline & \#1 & \#2 & $\# 3$ & $\# 4$ & $\# 5$ & \\
\hline 1 & 0,600 & 0,600 & 0,600 & 1,000 & 0,600 & 0,680 \\
\hline 2 & 0,600 & 1,000 & 1,000 & 1,000 & 0,600 & 0,840 \\
\hline 3 & 0,600 & 0,600 & 0,600 & 1,000 & 0,600 & 0,680 \\
\hline 4 & 0,429 & 1,000 & 0,600 & 0,600 & 0,429 & 0,611 \\
\hline 5 & 0,600 & 0,429 & 0,600 & 0,600 & 0,600 & 0,566 \\
\hline 6 & 0,429 & 0,600 & 0,429 & 0,600 & 0,600 & 0,531 \\
\hline 7 & 0,600 & 0,600 & 0,600 & 0,600 & 0,600 & 0,600 \\
\hline 8 & 0,429 & 0,600 & 0,600 & 0,429 & 0,429 & 0,497 \\
\hline 9,1 & 0,333 & 0,429 & 0,333 & 0,600 & 0,429 & 0,425 \\
\hline 9,2 & 0,429 & 0,429 & 0,600 & 0,600 & 0,429 & 0,497 \\
\hline 9,3 & 0,429 & 0,429 & 0,333 & 0,429 & 0,429 & 0,410 \\
\hline 10,1 & 0,600 & 0,600 & 1,000 & 1,000 & 1,000 & 0,840 \\
\hline 10,2 & 0,600 & 0,429 & 0,429 & 0,600 & 0,600 & 0,531 \\
\hline 10,3 & 0,600 & 0,333 & 0,600 & 0,600 & 0,429 & 0,512 \\
\hline 10,4 & 0,600 & 0,429 & 0,600 & 1,000 & 0,600 & 0,646 \\
\hline 10,5 & 0,600 & 1,000 & 1,000 & 0,600 & 0,600 & 0,760 \\
\hline
\end{tabular}

Tabla 6. Índice GRG- Evaluación Final de Indicadores de sostenibilidad. Dimensión Social. Fuente: elaboración propia, basada en Bhanot (2016a). 
EVALUACION DE INDICADORES DE SOSTENTABILIDAD - DIMENSION SOCIAL PLAN DE MEJORA CONTINUA 2017

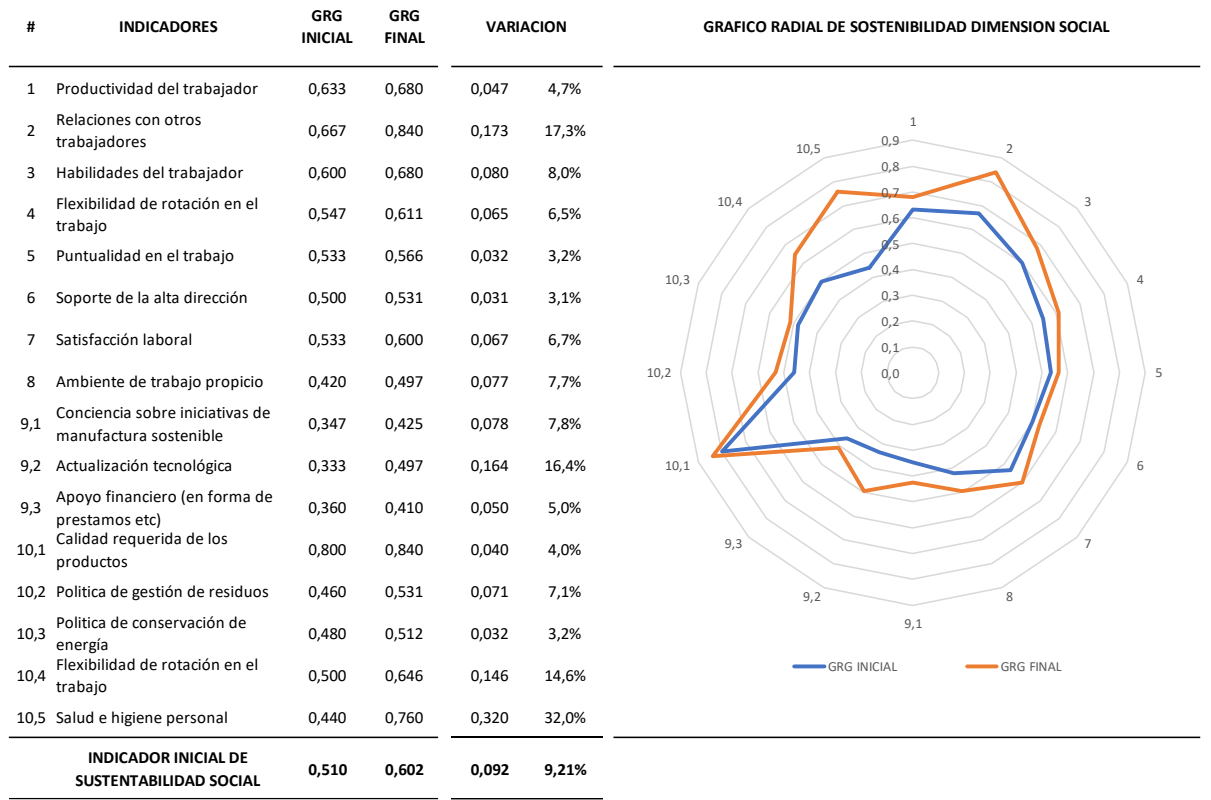

Tabla 7. Evaluación de Indicadores de Sostenibilidad -Dimensión Social.

Fuente: elaboración propia. 


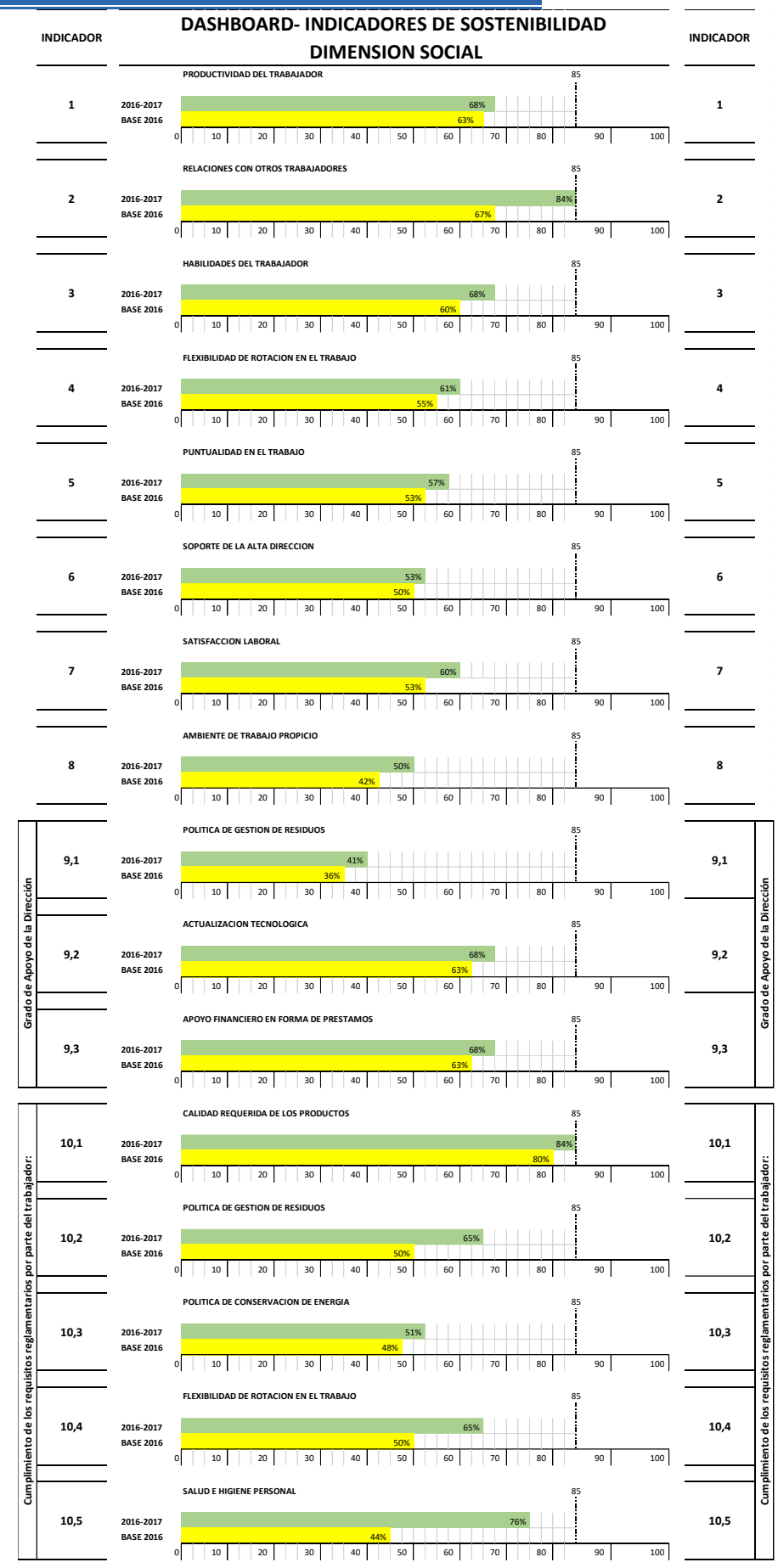

Tabla 8. Evaluación de Indicadores de sostenibilidad. Dimensión Social.

Fuente: elaboración propia.

PASO 8. Documentar y definir nuevos planes de mejora: al ser un ciclo de mejora continua se logra identificar opciones de mejora en aspectos como la conciencia sobre iniciativas de manufactura sostenible, ambiente de trabajo y la actualización tecnología, como los más relevantes y sobre los cuales se considerarían prioritaria para el siguiente plan de mejora.

En el diagnóstico de sostenibilidad final, un incremento global del 9,21\% de los indicadores de sostenibilidad social, evidenciando mejoras significativas, entre los que tenemos: relaciones con otros 


\section{${ }^{3}$ c) tecnología}

3C Tecnología (Edición 25) Vol.7- № 1

Marzo-junio '18, 61 - 78

Área de Innovación y Desarrollo, S.L.

ISSN: $2254-4143$

DOI: http://dx.doi.org/10.17993/3ctecno.2018.v7n1e25.61-78

trabajadores $(17,3 \%)$, actualización tecnológica $(16,4 \%)$ y flexibilidad de rotación en el trabajo $(14,6 \%)$.

En forma general se identifica que hay mejora, pero se requiere reforzar actividades específicas ya que algunos indicadores no alcanzar el 50\%, especial atención para los planes futuros de mejora continua, los relacionados con el grado de apoyo de la dirección, que son los que requieren mayor atención.

El clima laboral dentro de la empresa mejora y se establece buenas relaciones entre los colaboradores de esta, lo que se refleja con una mayor participación en los proyectos de mejora planteados. Si bien se observan mejoras significativas en el plan PHVA, se requiere implementar nuevos planes para poder alcanzar la meta de $85 \%$ en el próximo periodo de evaluación.

\section{CONCLUSIONES}

La metodología del Análisis Relacional Gris (Grey Relational Analysis) es adecuada para la evaluación de indicadores de sostenibilidad cualitativos de la dimensión social; ya que presenta facilidades para comparar las diferentes magnitudes en las que indicadores podrían estar expresadas, normaliza los resultados en valores comprendidos en el rango de 0 a 1 (o expresarlos en porcentajes) y permite un análisis homogéneo.

La selección adecuada de indicadores sostenibles permite un diagnóstico de los planes de mejora en procesos industriales, especialmente en procesos de manufactura. En la evaluación inicial y final, la empresa ha podido valorar la mejora continua de la sostenibilidad en la dimensión social.

Se verifica la aplicabilidad del ciclo de mejora continua de Deming, aplicado a la sostenibilidad y a la administración estrategia de las operaciones sustentables de manufactura.

La metodología GRA también es aplicable para la evaluación de las dimensiones económicas y ambientales las cuales tienen indicadores de tipo cualitativos en los procesos de manufactura de mecanizado, como son la tasa de remoción de material, rugosidad superficial, temperatura, etc., entre otros.

Los indicadores obtenidos, mediante técnicas de optimización complementarias, como el ANOVA y otros, permitirán definir los valores óptimos de las variables del proceso de mecanizado analizado.

\section{AGRADECIMIENTOS}

Los autores agradecen el apoyo de la Universitat Politècnica de València UPV, Fundación Carolina y Escuela Politécnica Nacional, para la realización del presente artículo por medio de la convocatoria 2017 y proyecto PIS 16-15 - PIS 16-22, ya que este artículo se ha desarrollado con su valiosa colaboración. 


\section{REFERENCIAS BIBLIOGRÁFICAS}

1. Bhanot, N., P.Venkateswara Rao, and S. G. Deshmukh. (2015). "Sustainability Assessment Framework for a Manufacturing Firm: An Exploratory Study." Journal of Practice Management 39(2):36-46.

2. Bhanot, N., P.Venkateswara Rao, and S. G. Deshmukh. (2016a). "An Assessment of Sustainability for Turning Process in an Automobile Firm." pp. 538-43 in Procedia CIRP, vol. 48. http://dx.doi.org/10.1016/j.procir.2016.03.024.

3. Bhanot, N., P.Venkateswara Rao, and S. G. Deshmukh. (2016b). "An Integrated Sustainability Assessment Framework: A Case of Turning Process." Clean Technologies and Environmental Policy 18(5):1475-1513.

4. Emprendices (2010). El ciclo PHVA y las Normas ISO 9000, Recuperado de: $<$ https://www.emprendices.co/el-ciclo-phva-y-las-normas-iso-9000/>.

5. GoConqr (2017). Desarrollo Sostenible. Recuperado de: <https://www.goconqr.com/p/3405661desarrollo-sostenible-flash card decks $>$.

6. Helu M. \& Dornfeld D. (2013). Principles of Green Manufacturing. En D.A. Dornfeld (ed.), Green Manufacturing: Fundamentals and Applications (111-113), Springer New York Heidelberg Dordrecht London. DOI: 10.1007/978-1-4419-6016-0/

7. Instituto Tecnológico y de Estudios Superiores de Monterrey (2013). ITESM Material ruta de la calidad. Recuperado de: <https://es.slideshare.net/manzanita64/material-ruta-de-la-calidad>.

8. Securityjeifer. (2010). Que es el ciclo PHVA. Recuperado de: <https://securityjeifer.wordpress.com/2010/09/01/\%C2\%BFque-es-el-ciclo-phva/phva/>.

9. United Nations (1987). Report of the World Commission on Environment and Development: Our Common Future. Recuperado de: $\langle$ www.un-documents.net/our-common-future.pdf $\rangle$.

10. United Nations (2015a). Objetivos de desarrollo sostenible. Recuperado de: <http://www.un.org/sustainabledevelopment/es/objetivos-de-desarrollo-sostenible/>.

11. United Nations (2015b). Se debe acelerara la implementación de los objetivos de desarrollo sostenible. Recuperado de: <http://www.un.org/sustainabledevelopment/es/2017/07/se-debeacelerar-la-implementacion-de-los-objetivos-de-desarrollo-sostenible/>.

12. Wang, S., X. Lu, X. X. Li, and W. D. Li. (2015). "A Systematic Approach of Process Planning and Scheduling Optimization for Sustainable Machining." Journal of Cleaner Production 87(C):914-29. 